\title{
Phase diagrams of the Bose-Fermi-Hubbard model: Hubbard operator approach
}

\author{
I.V. Stasyuk, T.S. Mysakovych, V.O.Krasnov \\ Institute for Condensed Matter Physics of the National Academy of Sciences of Ukraine, \\ 1 Svientsitskii Str., 79011 Lviv, Ukraine
}

Received March 3, 2010, in final form March 5, 2010

\begin{abstract}
The phase transitions in the Bose-Fermi-Hubbard model are investigated. The boson Green's function is calculated in the random phase approximation (RPA) and the formalism of the Hubbard operators is used. The regions of existence of the superfluid and Mott insulator phases are established and the $(\mu, t)$ (the boson chemical potential vs. hopping parameter) phase diagrams are built at different values of boson-fermion interaction constant (in the regimes of fixed chemical potential or fixed concentration of fermions). The effect of temperature change on this transition is analyzed and the phase diagrams in the $(T, \mu)$ plane are constructed. The role of thermal activation of the ion hopping is investigated by taking into account the temperature dependence of the transfer parameter. The reconstruction of the Mott-insulator lobes due to this effect is analyzed.
\end{abstract}

Key words: Bose-Fermi-Hubbard model, phase transitions, thermal activation

PACS: $03.75 . \mathrm{Lm}, 37.10 . \mathrm{Jk}, 67.85 . \mathrm{Pq}$

\section{Introduction}

There are a lot of papers devoted to the investigation of the properties of systems described by the Bose-Fermi-Hubbard model (BFHM) [1 5], which can be considered as a generalization of the Bose-Hubbard (BH) model [ 6$]$ in the case of the presence of fermions. One of the examples is an optical lattice. The Bose-Einstein (BE) condensation in boson-fermion mixtures (for example, ${ }^{6} \mathrm{Li}-{ }^{7} \mathrm{Li},{ }^{6} \mathrm{Li}-{ }^{87} \mathrm{Rb}$ ) confined in optical lattices has attracted great attention of physicists. The parameters of the BFHM which describes these objects can be tuned in experiments and results of theoretical calculations can be tested in practice. Different theoretical approaches were used to investigate the BFHM: mean field theory [2], strong coupling approach [3], density matrix renormalization group [4], quantum Monte Carlo [5] and others.

Another example of systems which can be described by the Bose-Fermi-Hubbard-type Hamiltonian are intercalated crystals (for example, $\mathrm{TiO}_{2}$ intercalated by $\mathrm{Li}$ ions). In such systems the impurity ions and electrons play the role of the bosons and fermions, respectively. A lattice gas type models are also used for the description of ionic conductors [7 -9]. In [10] the pseudospin-electron model of intercalation was formulated and thermodynamics of the model was investigated in the mean field approximation. It was shown that due to the presence of electrons the effective interaction between ions is generated and the character of this interaction depends on the filling of the electron band (when the chemical potential of the electrons is near the band edges, the first order phase transition between uniform phases or phase separation in the regime of the fixed electron concentration takes place; such an effect is important when constructing batteries [11]).

In this work we consider the BFHM and propose a method for calculation of correlation functions and average values of boson and fermion concentrations. The method is based on the introduction of Hubbard operators acting in the on-site basis of states and is similar to the composite fermion approach used in 2] (the composite fermions are formed by a fermion and bosons or bosonic holes) but is more general and universal. Introducing the on-site Hubbard operators we can employ a known technique developed for the calculation of a correlation (Green's) function built on these operators, for example, equation of motion method [12] or diagrammatic approach based on the corresponding Wick's theorem [13]. In this paper we use the first of them. As a first 
step, we calculate the boson Green's function in a particular case of immobile fermions and consider the condition of instability of normal phase with respect to the appearance of the BE condensate. Constructing the phase diagrams in the (temperature-chemical potential of bosons) and (hopping parameter of bosons - chemical potential of bosons) planes, we analyse the effect of fermions on the transition to the superfluid (SF) phase at different relations between the model parameters. We also illustrate the ground state diagrams of the model in the cases of repulsion or attraction between bosons and fermions.

\section{The random phase approximation}

Mixtures of ultracold bosons and spin-polarized fermions in optical lattices are well described by the Bose-Fermi- Hubbard Hamiltonian [1]

$$
H=-\sum_{i j} t_{i j} b_{i}^{+} b_{j}-\sum_{i j} t_{i j}^{\prime} f_{i}^{+} f_{j}+\frac{U}{2} \sum_{i} n_{i}^{b}\left(n_{i}^{b}-1\right)+U^{\prime} \sum_{i} n_{i}^{b} n_{i}^{\mathrm{f}}-\mu \sum_{i} b_{i}^{+} b_{i}-\mu^{\prime} \sum_{i} f_{i}^{+} f_{i} .
$$

The first (second) term describes nearest neighbor boson (fermion) hopping with $t\left(t^{\prime}\right)$ denoting the tunneling amplitude of bosons (fermions). The third and forth terms are on-site boson-boson and boson-fermion interactions, respectively. The last two terms involve the chemical potentials of the bosons $\mu$ and fermions $\mu^{\prime}$, that are introduced to change the mean concentrations of these particles.

The first step is to introduce the following on-site basis

$$
\left(n_{i}^{b}=n ; n_{i}^{\mathrm{f}}=0\right) \equiv|n, i\rangle ; \quad\left(n_{i}^{b}=n ; n_{i}^{\mathrm{f}}=1\right) \equiv|\tilde{n}, i\rangle,
$$

where $n_{i}^{b}$ or $n_{i}^{\mathrm{f}}$ is the number of the bosons or fermions on site $i$, respectively. Now we can introduce the Hubbard operators $X_{i}^{n m}=|n, i\rangle\left\langle m, i\left|, X_{i}^{\tilde{n} \tilde{m}}=\right| \tilde{n}, i\right\rangle\langle\tilde{m}, i|$, etc. As a result, the Bose- and Fermi-operators of creation and annihilation can be written as

$$
\begin{aligned}
b_{i} & =\sum_{n} \sqrt{n+1} X_{i}^{n, n+1}+\sum_{n} \sqrt{\tilde{n}+1} X_{i}^{\tilde{n}, \tilde{n}+1}, \\
b_{i}^{+} & =\sum_{n} \sqrt{n+1} X_{i}^{n+1, n}+\sum_{n} \sqrt{\tilde{n}+1} X_{i}^{\tilde{n}+1, \tilde{n}}, \\
n_{i}^{b} & =\sum_{n} n X_{i}^{n n}+\sum_{\tilde{n}} \tilde{n} X_{i}^{\tilde{n} \tilde{n}}, \quad n_{i}^{\mathrm{f}}=\sum_{n} X_{i}^{\tilde{n} \tilde{n}}, \\
a_{i} & =\sum_{n} X_{i}^{n, \tilde{n}}, \quad a_{i}^{+}=\sum_{n} X_{i}^{\tilde{n}, n} .
\end{aligned}
$$

The Hamiltonian takes in this representation the following form

$$
\begin{aligned}
H & =H_{0}+H_{1}^{b}+H_{1}^{\mathrm{f}}, \\
H_{0} & =\sum_{i n} \lambda_{n} X_{i}^{n n}+\sum_{i \tilde{n}} \lambda_{\tilde{n}} X_{i}^{\tilde{n} \tilde{n}} \\
\lambda_{n} & =\frac{U}{2} n(n-1)-\mu n, \quad \lambda_{\tilde{n}}=\frac{U}{2} \tilde{n}(\tilde{n}-1)-\mu \tilde{n}-\mu^{\prime}+U^{\prime} \tilde{n} \\
H_{1}^{b} & =\sum_{i j} t_{i j} b_{i}^{+} b_{j}, \quad H_{1}^{\mathrm{f}}=\sum_{i j} t_{i j}^{\prime} a_{i}^{+} a_{j} .
\end{aligned}
$$

In this case the on-site part $H_{0}$ becomes diagonal and can play the role of an unperturbed part of the Hamiltonian, when expansion in terms of the hopping parameters in such a type of models is used [14].

In this work we restrict ourselves to the equation of motion method. To find the two-time Green's function $G_{i j}\left(t-t^{\prime}\right)=\left\langle\left\langle b_{i}(t) \mid b_{j}^{+}\left(t^{\prime}\right)\right\rangle\right\rangle$ we write the equation for Fourier transform of its 
constituent part

$$
\omega\left\langle\left\langle X_{l}^{m, m+1} \mid X_{p}^{r+1, r}\right\rangle\right\rangle=\frac{1}{2 \pi} \delta_{l p} \delta_{m r}\left\langle X_{l}^{m r}-X_{l}^{r+1, m+1}\right\rangle+\left\langle\left\langle\left[X_{l}^{m, m+1}, \widehat{H}\right] \mid X_{p}^{r+1, r}\right\rangle\right\rangle .
$$

We use the random phase approximation (which is an analogy to the Hubbard-I approximation in the case of the fermionic Hubbard model and is a basic one for the usual Bose-Hubbard model, see [15, 16]) and perform the following decoupling:

$$
\begin{aligned}
\left\langle\left\langle\left(X_{l}^{m, m}-X_{l}^{m+1, m+1}\right) b_{j} \mid X_{p}^{r+1, r}\right\rangle\right\rangle & \approx\left\langle X^{m, m}-X^{m+1, m+1}\right\rangle\left\langle\left\langle b_{j} \mid X_{p}^{r+1, r}\right\rangle\right\rangle, \\
\left\langle\left\langle b_{i}^{+} X^{m, m+2} \mid X_{r}^{r+1, r}\right\rangle\right\rangle & \approx 0 .
\end{aligned}
$$

Such an approximation is valid in the case when $\left\langle b_{i}\right\rangle=0$ that corresponds to the normal (so-called Mott-insulator(MI)) phase. The commutators $\left[X^{m n}, H\right]$ become

$$
\begin{aligned}
& {\left[X_{l}^{m, m+1}, \widehat{H}\right]=\Delta_{m} X_{l}^{m, m+1}+\sum_{j} t_{l j} \sqrt{m+1} Q_{m} b_{j}-\sum_{j} t_{l j}^{\prime} X_{l}^{\tilde{m}, m+1} a_{j}+\sum_{i} t_{i l}^{\prime} a_{i}^{+} X_{l}^{m, \tilde{m}+1},} \\
& {\left[X_{l}^{\tilde{m}, \tilde{m}+1}, \widehat{H}\right]=\Delta_{\tilde{m}} X_{l}^{\tilde{m}, \tilde{m}+1}+\sum_{j} t_{l j} \sqrt{\tilde{m}+1} Q_{\tilde{m}} b_{j}+\sum_{j} t_{l j}^{\prime} X_{l}^{\tilde{m}, m+1} a_{j}-\sum_{i} t_{i l}^{\prime} a_{i}^{+} X_{l}^{m, \tilde{m}+1},}
\end{aligned}
$$

where $\Delta_{m}=\lambda_{m+1}-\lambda_{m}\left(\Delta_{\tilde{m}}=\lambda_{\tilde{m}+1}-\lambda_{\tilde{m}}\right)$ is a distance between on-site energy levels, and $Q_{m}=$ $\left\langle X^{m, m}-X^{m+1, m+1}\right\rangle\left(Q_{\tilde{m}}=\left\langle X^{\tilde{m}, \tilde{m}}-X^{\tilde{m}+1, \tilde{m}+1}\right\rangle\right)$ is a difference between the mean occupancy of these levels.

In what follows we consider the case of localized fermions with $t_{i j}^{\prime}=0$. Using the relations (3) we can write the equation for the Green's function $\left\langle\left\langle b_{j} \mid X_{p}^{r, r+1}\right\rangle\right\rangle$

$$
\begin{aligned}
\left\langle\left\langle b_{l} \mid X_{p}^{r, r+1}\right\rangle\right\rangle= & \sum_{m} \frac{\hbar}{2 \pi} \delta_{l p} \delta_{m r} \sqrt{m+1} \frac{Q_{m}}{\omega-\Delta_{m}}+\sum_{m} \frac{Q_{m}(m+1)}{\hbar \omega-\Delta_{m}} \sum_{j} t_{l j}\left\langle\left\langle b_{j} \mid X_{p}^{r+1, r}\right\rangle\right\rangle \\
& +\sum_{\tilde{m}} \frac{Q_{\tilde{m}}(\tilde{m}+1)}{\omega-\Delta_{\tilde{m}}} \sum_{j} t_{l j}\left\langle\left\langle b_{j} \mid X_{p}^{r+1, r}\right\rangle\right\rangle .
\end{aligned}
$$

The equation for the function $\left\langle\left\langle b_{l} \mid X_{p}^{\tilde{r}, \tilde{r}+1}\right\rangle\right\rangle$ has a similar form. We pass to $\boldsymbol{k}$-representation and introduce the unperturbed Green's function

$$
g^{0}(\omega)=\sum_{m}\left[\frac{Q_{m}(m+1)}{\omega-\Delta_{m}}+\frac{Q_{\tilde{m}}(\tilde{m}+1)}{\omega-\Delta_{\tilde{m}}}\right] .
$$

As a result, the expression for the Green's function $G_{\boldsymbol{k}}(\omega)$ can be obtained

$$
G_{\boldsymbol{k}}(\omega)=\frac{1}{2 \pi} \frac{g^{0}(\omega)}{1-g^{0}(\omega) t_{\boldsymbol{k}}} .
$$

The obtained expression (11) for the bosonic Green's function allows us to investigate the effect of the fermions on stability of the normal phase and to establish the conditions of the appearance of Bose-Einstein condensate in the mixed boson-fermion systems. In the next section we build the corresponding phase diagrams.

\section{Phase diagrams}

First of all, we can build the diagrams of the ground state of the system in the plane $\left(\mu^{\prime}, \mu\right)$ using the unperturbed Hamiltonian $H_{0}$ (in this case the averages of the Hubbard operators are given by $\left\langle X^{m m}\right\rangle=\frac{1}{Z} \mathrm{e}^{-\beta \lambda_{m}},\left\langle X^{\tilde{m} \tilde{m}}\right\rangle=\frac{1}{Z} \mathrm{e}^{-\beta \lambda_{\tilde{m}}}$, where $\left.Z=\sum_{m} \mathrm{e}^{-\beta \lambda_{m}}+\sum_{\tilde{m}} \mathrm{e}^{-\beta \lambda_{\tilde{m}}}\right)$.

In figure 1 and figure 2, we show the ground state diagrams $(T=0)$ at different values of $U$ and $U^{\prime}$ parameters. It is seen that in comparison with the Bose-Hubbard model (where the vertical 
lines are present only) new regions appear. The inclined lines separate the states $|m\rangle$ and $|\tilde{n}\rangle$ with different boson and fermion numbers. General shape of the diagrams depends on the sign of the boson-fermion interaction constant. Such an effect was also pointed out in [2] in the framework of the composite fermion picture.
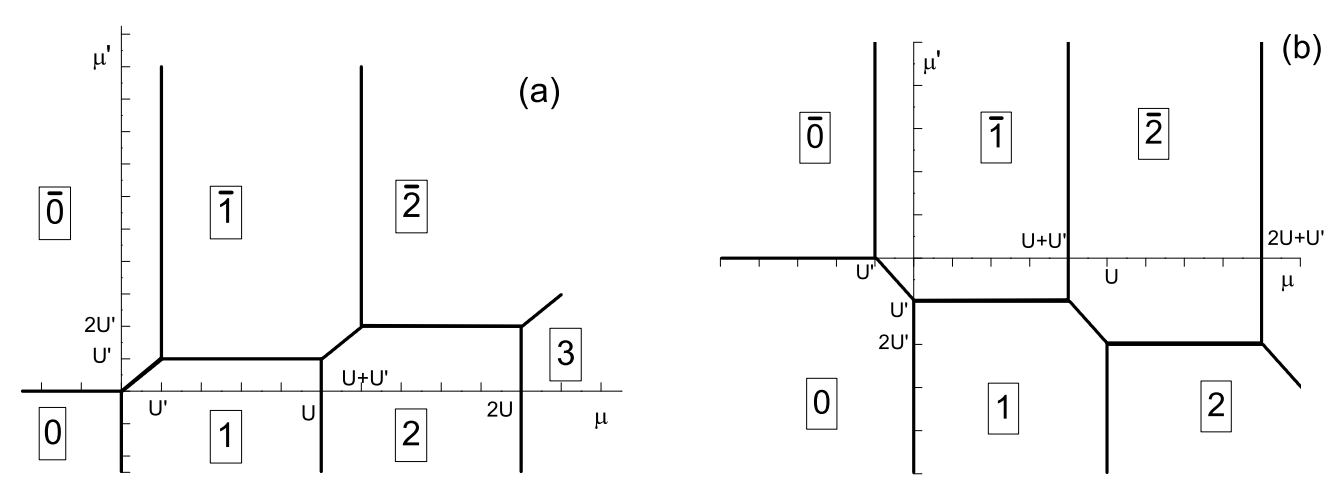

Figure 1. Ground state diagrams for $0<U^{\prime}<U$ (a) and $-U<U^{\prime}<0$ (b).
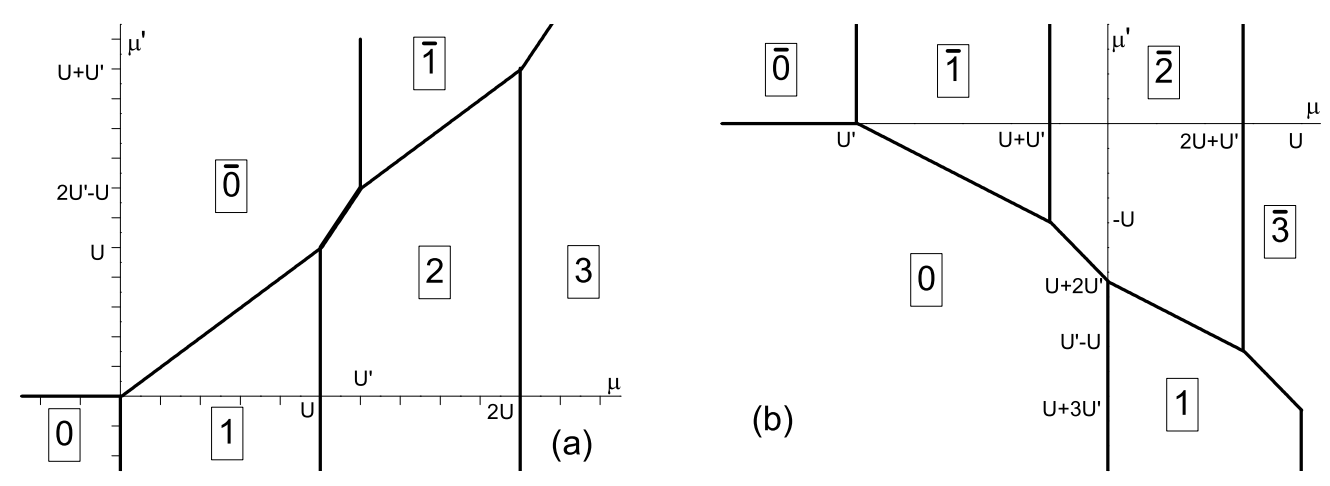

Figure 2. Ground state diagrams for $U<U^{\prime}<2 U$ (a) and $-2 U<U^{\prime}<-U<0$ (b).

The phase transition from the Mott-insulator to SF phase is characterized by divergence of the Green's function $G_{\boldsymbol{k}=0}(\omega=0) \rightarrow \infty$ that leads to the equation

$$
1=t_{0} \sum_{m}\left(\frac{Q_{m}(m+1)}{\Delta_{m}}+\frac{Q_{\tilde{m}}(\tilde{m}+1)}{\Delta_{\tilde{m}}}\right) .
$$

This condition in the case $t>0$ (considered in this paper) is fulfilled at the center $\boldsymbol{k}=0$ of the Brillouin zone (an uniform SF phase case). We should note that at $T \rightarrow 0$ the equation (12) corresponds to that obtained in [2] in the framework of the mean field theory.

In figure 3, 4 and figure 5, we show the $(W, \mu)$ phase diagrams (here $W$ is a half width of the initial bosonic energy band, $-W<t_{k}<W$ ) in the cases of the constant fermion concentration or fermion chemical potential. Similarly to the pure Bose-Hubbard model, the regions where the MI phase is stable have the form of characteristic lobes, but, in general, the shape of such a phase boundary is more complicated. In the special case $\frac{U^{\prime}}{U}=0,5$ the twice lesser multiplicity with respect to the $\frac{\mu}{U}$ ratio is observed (see figure $3(\mathrm{a})$ ).

In figure 4 and figure 5 a, we give the $(W, \mu)$ phase diagrams for different values of on-site interaction between bosons and fermions $U^{\prime}$ and average numbers of fermions $n^{\mathrm{f}}$. In the limit 

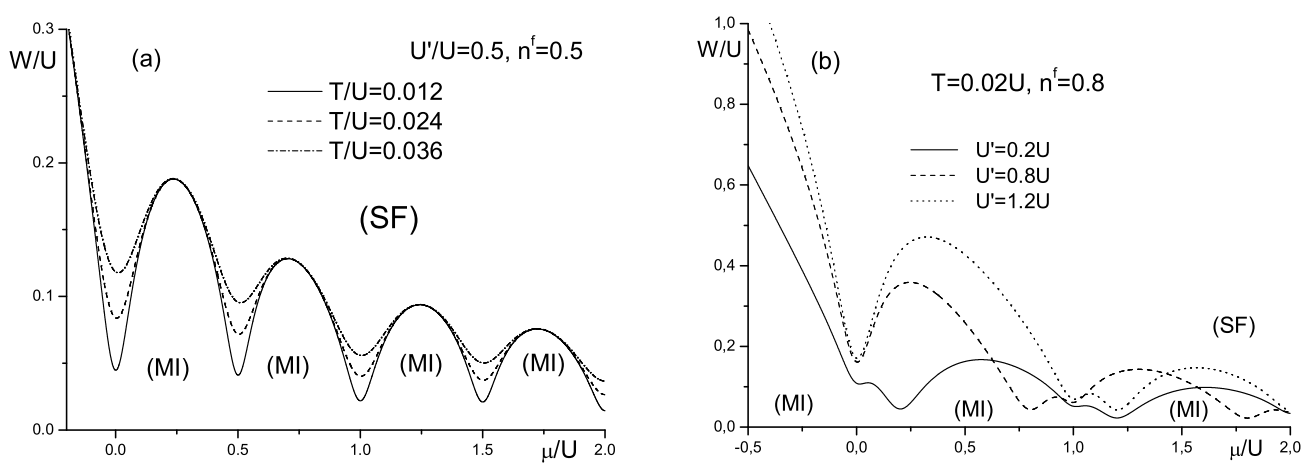

Figure 3. $(W, \mu)$ phase diagrams. a) The case of different values of temperature at $U^{\prime} / U=$ $0.5, n^{\mathrm{f}}=0.5$. b) The case of different values of boson-fermion interaction parameter at $T / U=$ $0.02, n^{\mathrm{f}}=0.8$.
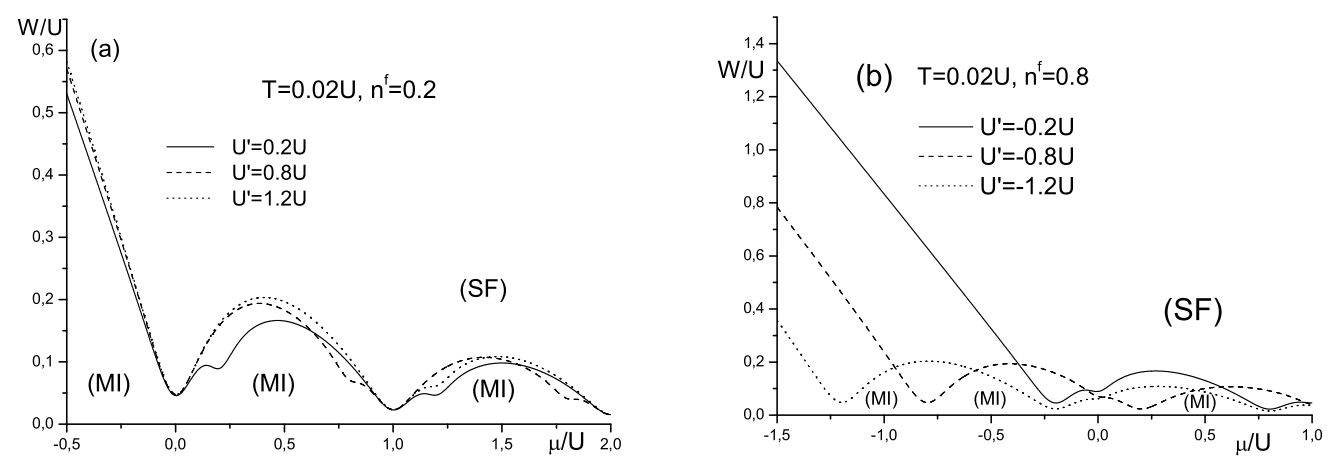

Figure 4. $(W, \mu)$ phase diagram for different values of $U^{\prime}$ and $n^{\mathrm{f}}$. a) $\left.n^{\mathrm{f}}=0.2, \mathrm{~b}\right) n^{\mathrm{f}}=0.8$.
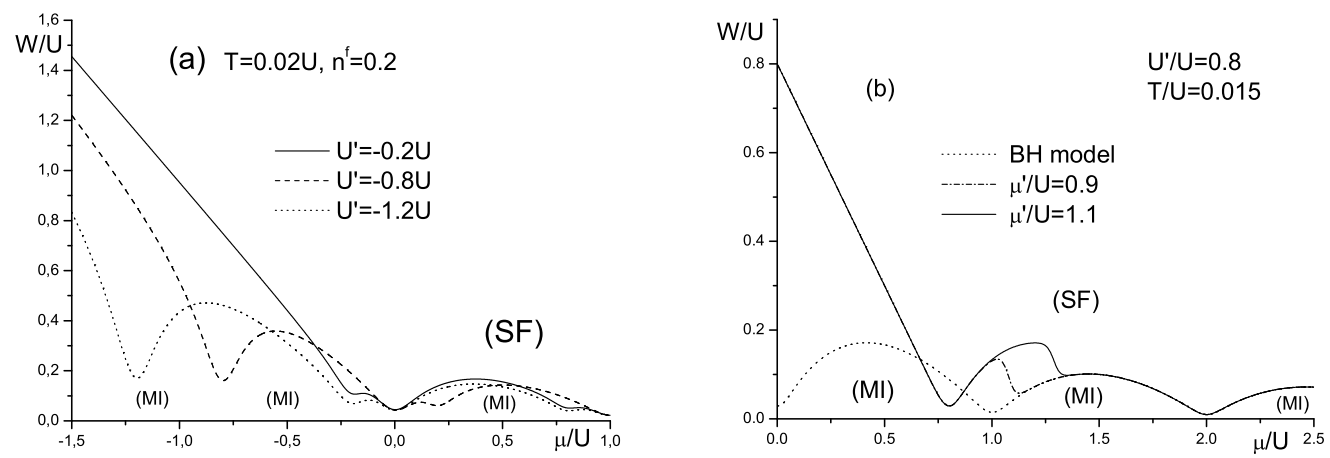

Figure 5. $(W, \mu)$ phase diagrams. a) The case of constant concentration of fermions at different values of $U^{\prime}$, b) The case of constant chemical potential of fermions.

$T \rightarrow 0$ the similar phase diagrams were obtained in [2]. For comparison, the diagrams are shown, that correspond to the cases of repulsion $\left(U^{\prime}>0\right)$ and attraction $\left(U^{\prime}<0\right)$ between bosons and fermions. It can be seen that in the first case the BE-condensate appears in the presence of fermions at higher values of boson tunneling constant; in the second case the opposite effect takes place. 
We do not consider in this work the appearance of a charge modulation phase, this is the task for future investigations.

The presence of more than one Mott-insulator lobe in the region $n<\mu / U<n+1$ is a characteristic feature of the Bose-Fermi-Hubbard model in comparison with the Bose-Hubbard model (in the latter case only one Mott-insulator lobe is present in the mentioned region). The positions of the additional lobes depend on the strength of the boson-fermion interaction only and are consisted with the location of vertical lines on the ground state diagrams (figures [1 and 2), where the instability with respect to the appearance of BE-condensate at $T=0$ takes place. The heights of these lobes depend on the fermion concentration.

In the regime of a constant chemical potential of fermions (see figure $5 \mathrm{~b}$ ) the reconstruction of the MI lobe takes place in the region of chemical potential $\mu$ values, which corresponds to intersection of $\mu^{\prime}=$ const line with inclined boundary lines separating the ground state regions with the different on-site boson and fermion occupation (see figures 11and22). On the left hand side of this intersection the BE-condensate appears in the limit $T \rightarrow 0$ at $\mu \sim U^{\prime}, U+U^{\prime}, 2 U+U^{\prime}, \ldots$ while on the right hand side it appears at $\mu=0, U, 2 U, \ldots$
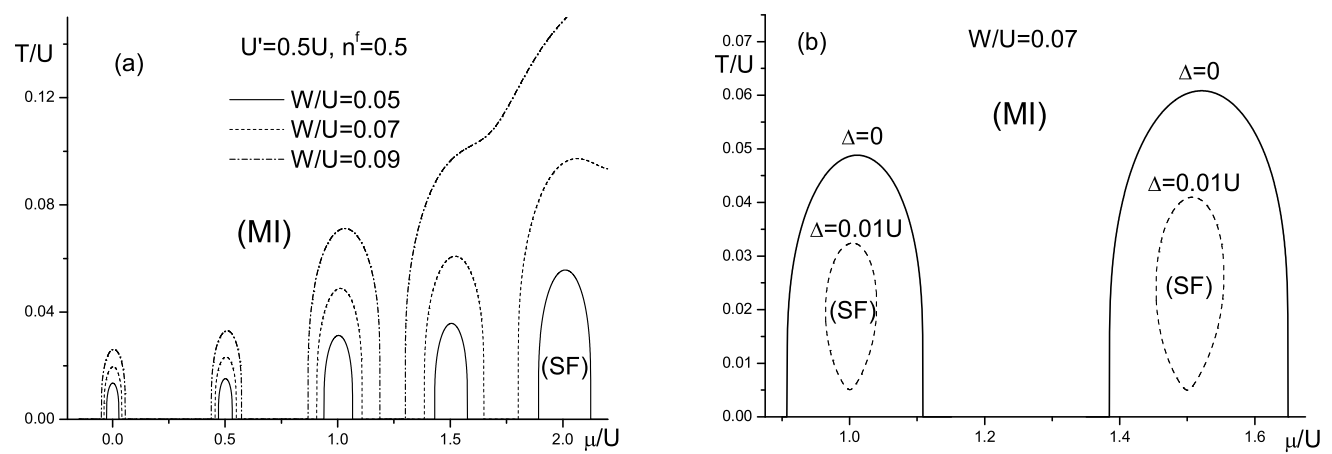

Figure 6. $(T, \mu)$ phase diagrams at $U^{\prime}=0.5 U, n^{\mathrm{f}}=0.5$. a) $\Delta=0$, b) $\Delta=0.01 U$.

The $(T, \mu)$ phase diagram is shown in figure [6. In comparison with pure Bose-Hubbard model (see e.g. [16]) the critical temperatures of transition into SF phase at the presence of fermions are lower (besides that, new regions with SF phase appear; at $U^{\prime} / U=0,5$ they are located around half-integer values of the $\mu / U$ ratio). We also depicted the $(T, \mu)$ diagram for the case of thermal activation of boson hopping. Such a possibility was considered in [16], having in mind the application of BH-model to the ionic conductors. We have rewritten the parameter of the bosonic (ionic in the case of ionic conductors) hopping in the form: $t=t_{0} \exp (-\beta \Delta)$ that can be obtained as a result of renormalization due to the interaction with phonons. Thermal activation leads to the increase of the Mott-insulator phase region. At the increase of the activation energy the region of the existence of the SF phase narrows and then this phase disappears. At the fixed value of the boson chemical potential (and fixed value of the fermion concentration) there are two critical temperatures at which the phase transition from the Mott insulator to the SF phase takes place. A similar effect is also present in the case of the Bose-Hubbard model; as was supposed in [16] the lower critical temperature can be related to the transition to the superionic state in the superionic crystals.

\section{Conclusions}

In this work we have investigated phase transitions in the BFHM at finite temperature using the on-site Hubbard operators formalism. We have restricted ourselves to the consideration of the case of localized fermions $\left(t_{\mathrm{f}}=0\right)$ and obtained the expression for boson Green's function in the RPA. The instability of normal phase with respect to the transition into the superfluid state at different 
values of the fermion concentrations and boson-fermion interaction constant is investigated. We have also considered the effect of the temperature change on this phase transition. The ground state diagrams in the plane (chemical potential of electrons - chemical potential of bosons) are built and regions of stability of the fermion composite type states are separated. Subsequently we plan to take into account the fermionic hopping and study its effect on the transition into SF phase in the framework of the developed scheme.

\title{
References
}

1. Albus A., Illuminati F., Eisert J., Phys. Rev. A, 2003, 68, 023606.

2. Lewenstein M., Santos L., Baranov M.A., Fehrmann H., Phys. Rev. Lett., 2004, 92, 050401.

3. Iskin M., Freericks J.K., Phys. Rev. A, 2009, 80, 053623.

4. Mering A., Fleischhauer M. Preprint arXiv:0903.5226.

5. Hébert F., Batrouni G.G., Roy X., Rousseau V.G., Phys. Rev. B, 2008, 78, 184505.

6. Fisher M.P.A., Weichman P.B., Grinstein G., Fisher D.S., Phys. Rev. B, 1989, 40, 546.

7. Mahan G.D, Phys. Rev. B, 1976, 14, 780.

8. Tomoyose T., J. Phys. Soc. Jpn., 1997, 66, 2383.

9. Stasyuk I.V., Dulepa I.R., Condens. Matter Phys., 2007, 10, 259.

10. Mysakovych T.S., Krasnov V.O., Stasyuk I.V., Condens. Matter Phys., 2008, 11, 663.

11. Wagemaker M., van de Krol R., Kentgens A.P.M., van Well A.A., Mulder F.M., J. Am. Chem. Soc., 2001, 123, 11454.

12. Hubbard J. P. R. Soc. Lond. A Mat., 1965, 285, 542.

13. Slobodyan P.M., Stasyuk I.V., Teor. Mat. Phys., 1974, 19, 423.

14. Stasyuk I.V., Shvaika A.M. Condens. Matter Phys., 1994, 3, 134.

15. Konabe S., Nikuni T., Nakamura M., Phys. Rev. A, 2006, 73, 033621-12.

16. Stasyuk I.V., Mysakovych T.S. Condens. Matter Phys., 2009, 12, 539.

\section{Фазові діаграми моделі Бозе-Фермі-Габбарда: метод операторів Габбарда}

\author{
І.В. Стасюк, Т.С. Мисакович, В.О. Краснов \\ Інститут фізики конденсованих систем НАН України, 79011 Львів, вул. Свєнціцького, 1
}

Досліджено фазові переходи в моделі Бозе-Фермі-Габбарда. Бозонна функція Гріна розрахована в наближенні хаотичних фаз з використанням формалізму операторів Габбарда. Встановлено області існування надплинної фази та фази Моттівського діелектрика і побудовано фазові діаграми в площині $(\mu, t)$ (хімічний потенціал бозонів-параметр перескоку) при різних значеннях константи бозонферміонної взаємодії (у режимах фіксованого хімічного потенціалу чи концентрації ферміонів). Проаналізовано вплив зміни температури на фазові переходи та побудовано фазові діаграми в площині $(T, \mu)$. Досліджено роль термічної активації перескоку частинок при врахуванні температурної залежності параметра перескоку. Проаналізовано перебудову фазових діаграм у цьому випадку.

Ключові слова: модель Бозе-Фермі-Габбарда, фазові переходи, термічна активація 
